\title{
Sub-2 nm Ir Nanoclusters Immobilized on Mesoporous Nitrogen-Doped Carbons as Efficient Catalysts for Selective Hydrogenation
}

Lin-Wei Chen,${ }^{\dagger}$ Lei Tong, ${ }^{\dagger}$ Hang Nan,${ }^{\dagger}$ Sheng-Qi Chu,${ }^{\dagger}$ and Hai-Wei Liang* ${ }^{\dagger}$

†Hefei National Laboratory for Physical Sciences at the Microscale, Department of Chemistry, University of Science and Technology of China, Hefei, 230026, China.

Institute of High Energy Physics, Chinese Academy of Sciences, Beijing, 100049, China

*E-mail: hwliang@ustc.edu.cn

\section{Supplementary figures}

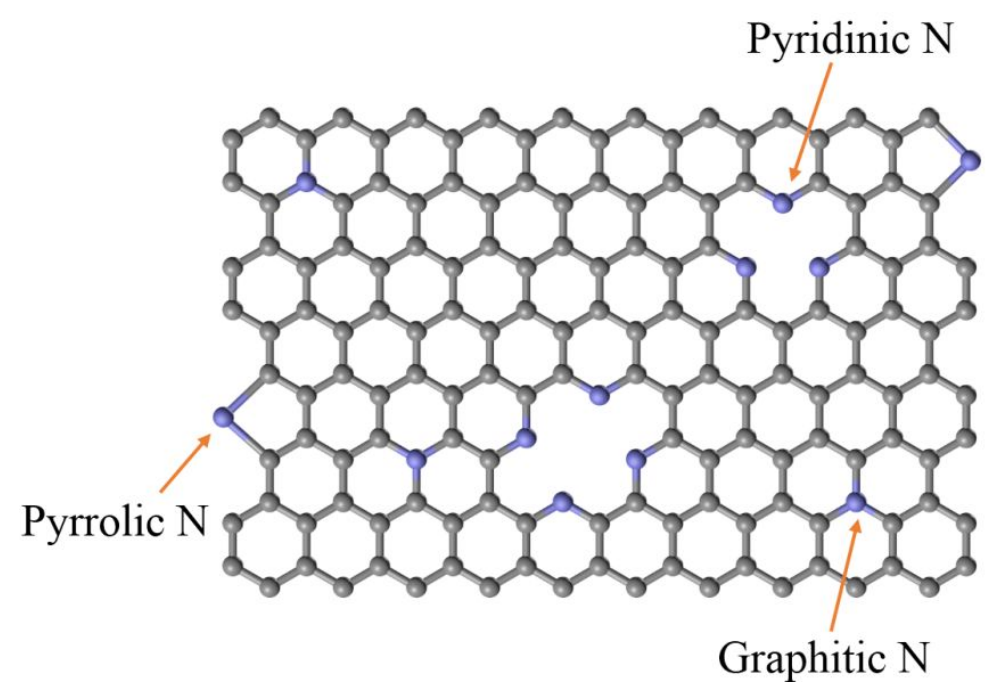

Figure S1. Different types of doped nitrogen atoms in the carbon materials. 


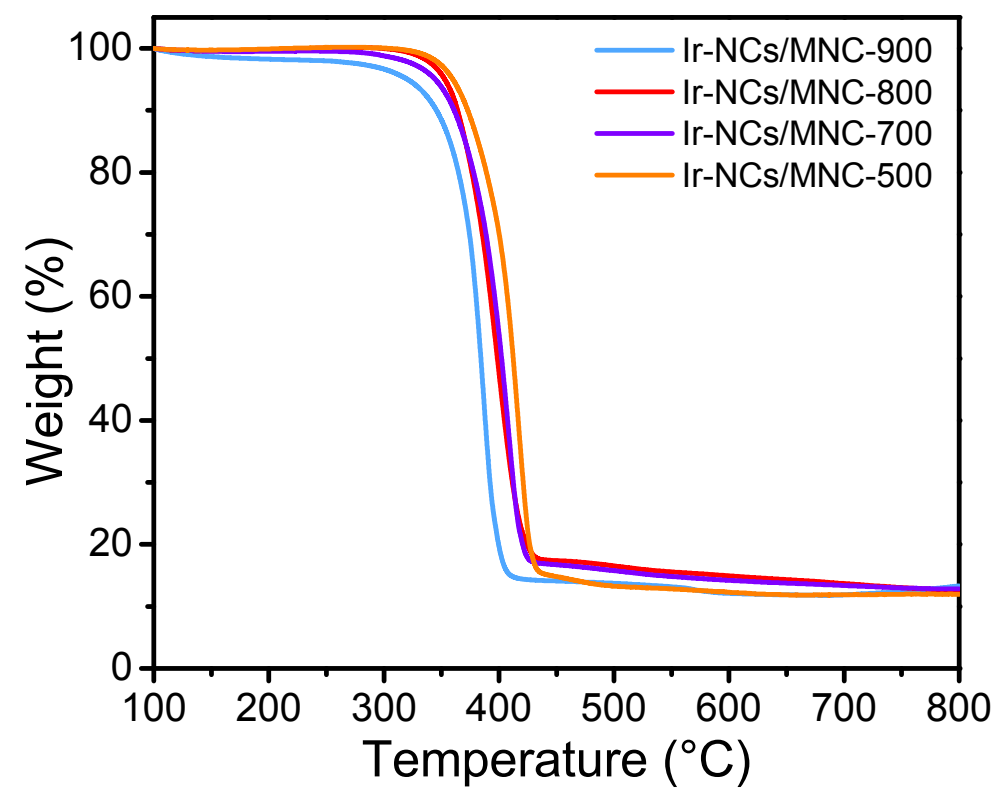

Figure S2. TGA curves of the Ir-NCs/MNC catalysts in $\mathrm{O}_{2}$ atmosphere. 

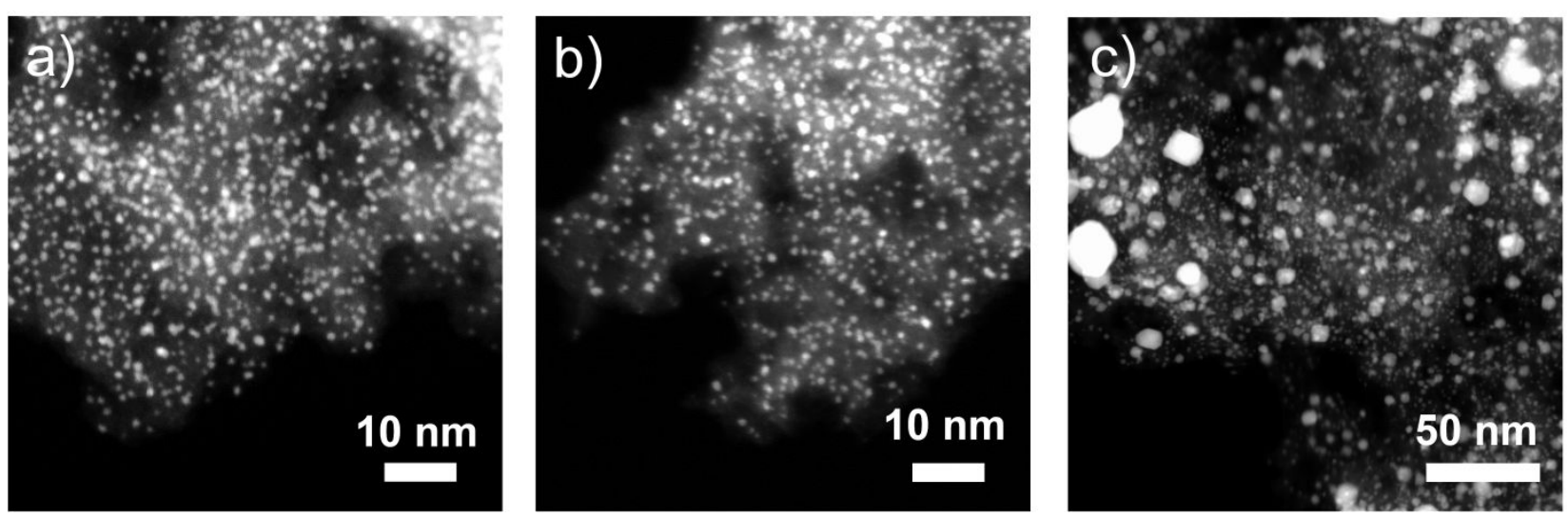

Figure S3. HAADF-STEM images of a) Ir-NCs/MNC-500, b)Ir-NCs/MNC-700, and c) IrNCs/MNC-900. 

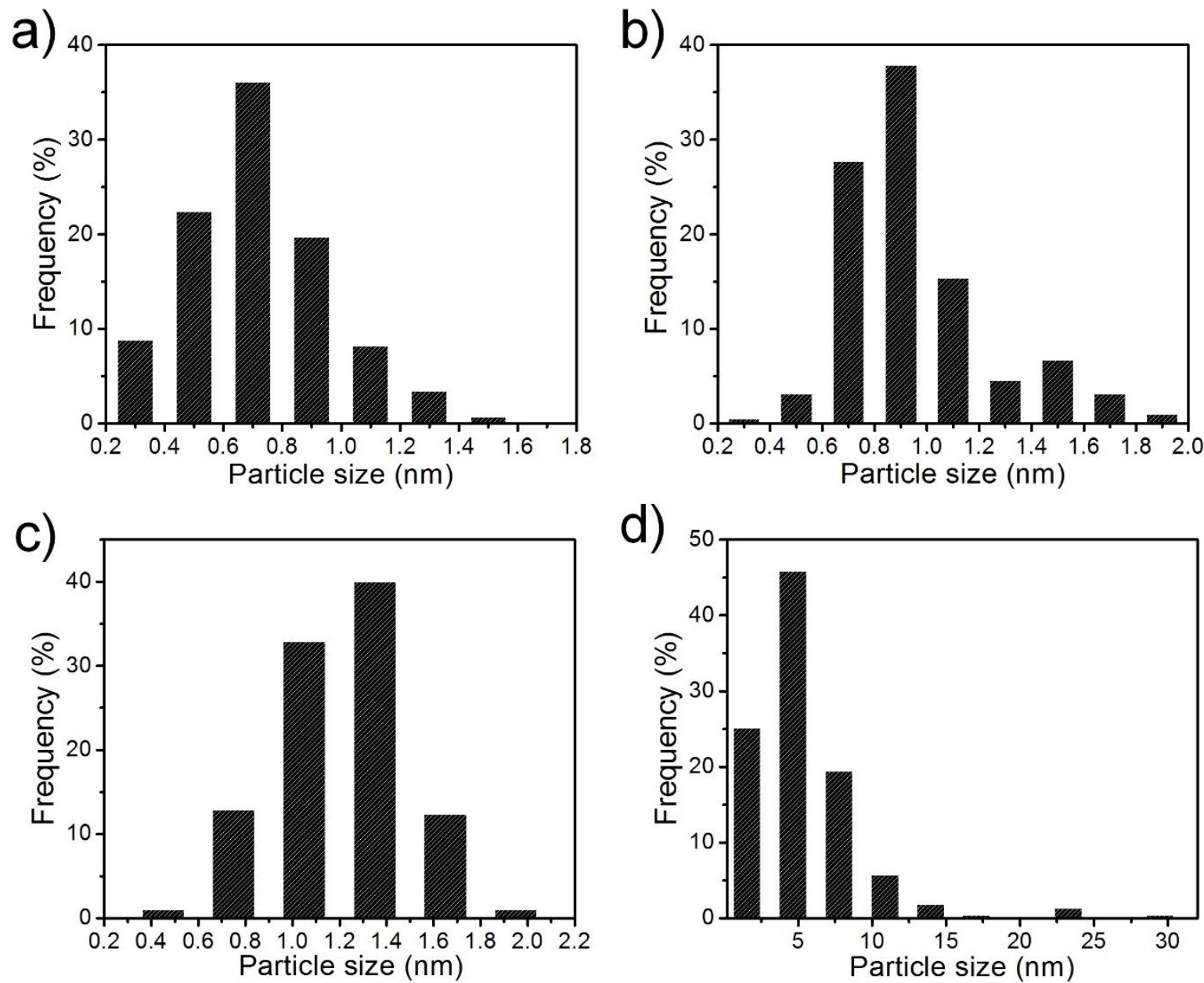

Figure S4. Size distributions of a) Ir-NCs/MNC-500, b) Ir-NCs/MNC-700, c) Ir-NCs/MNC-800, and d) $\mathrm{Ir}-\mathrm{NCs} / \mathrm{MNC}-900$. 


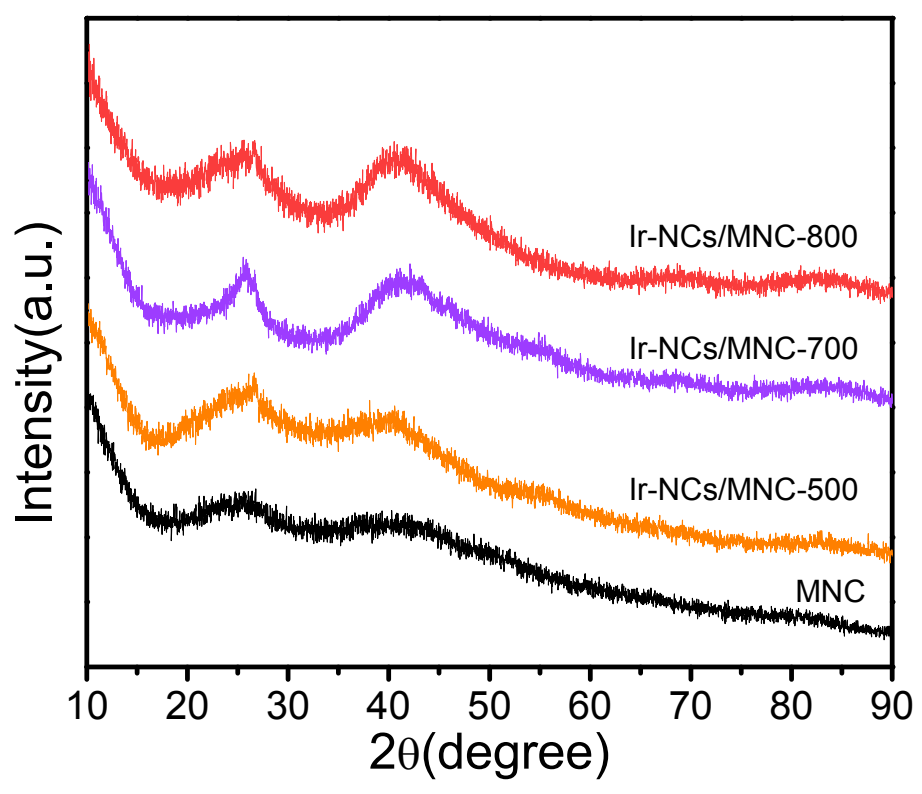

Figure S5. XRD patterns of the MNC support and the Ir-NCs/MNC catalysts prepared at 500, 700, and $800{ }^{\circ} \mathrm{C}$. 

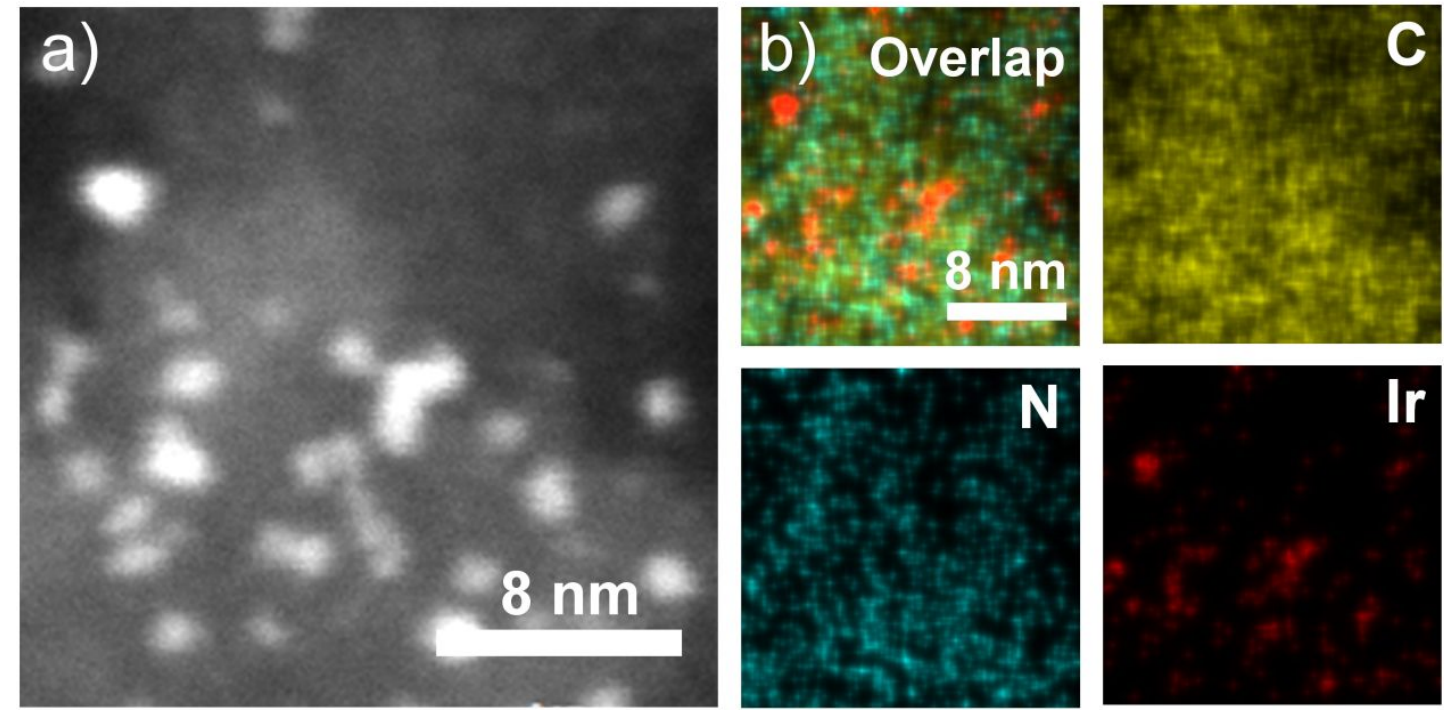

Ir

Figure S6. STEM-EDS elemental mapping of Ir-NCs/MNC-800. 
a)

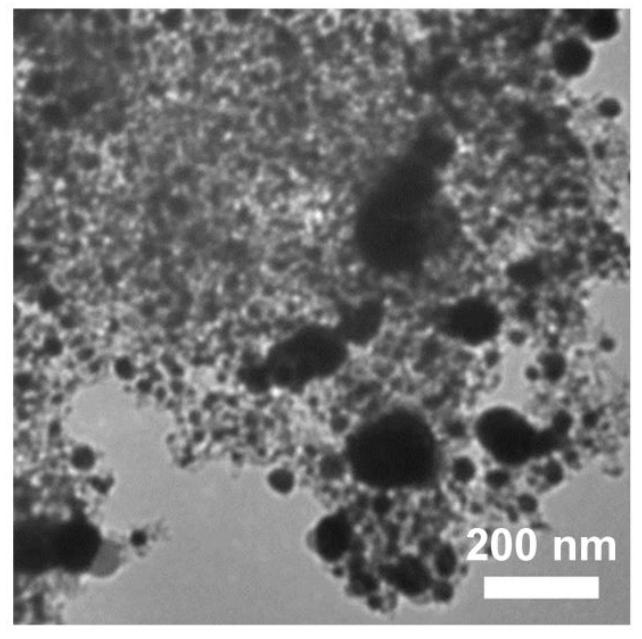

b)

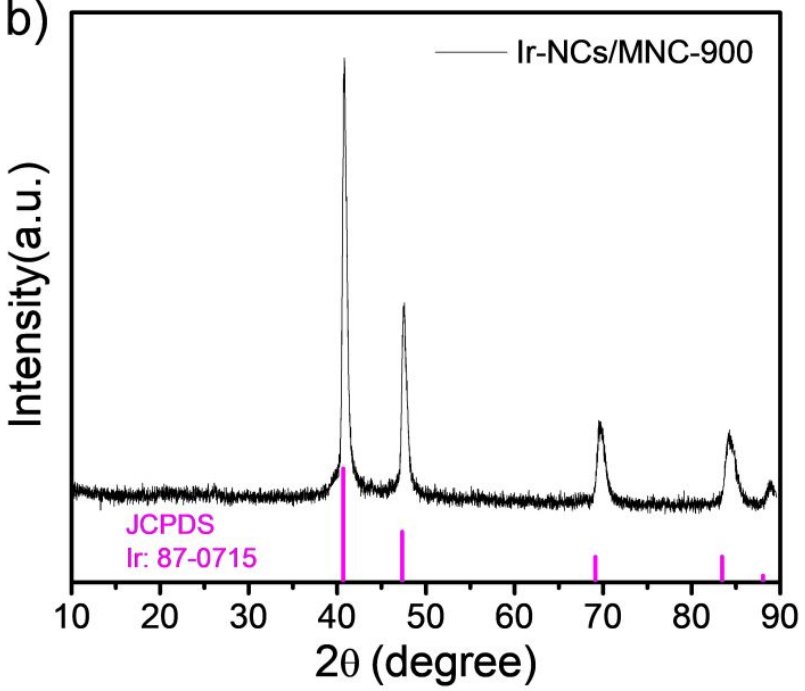

Figure S7. a) TEM image and b) XRD pattern of Ir-NCs/MNC-900. 
a)

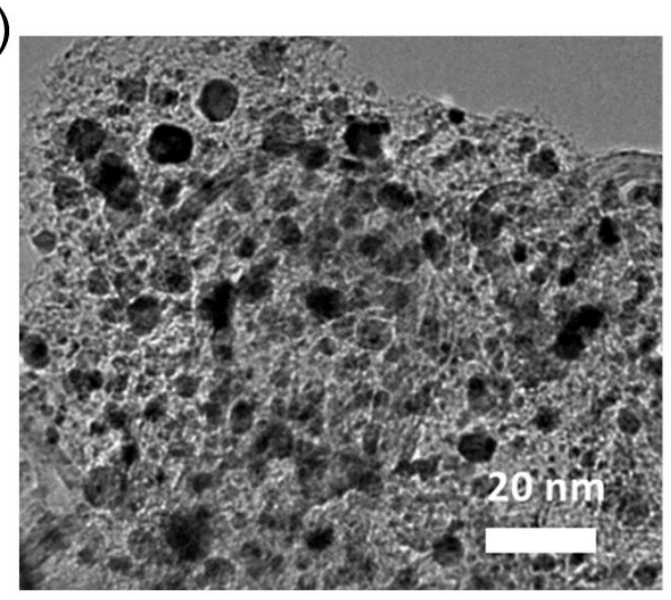

b)

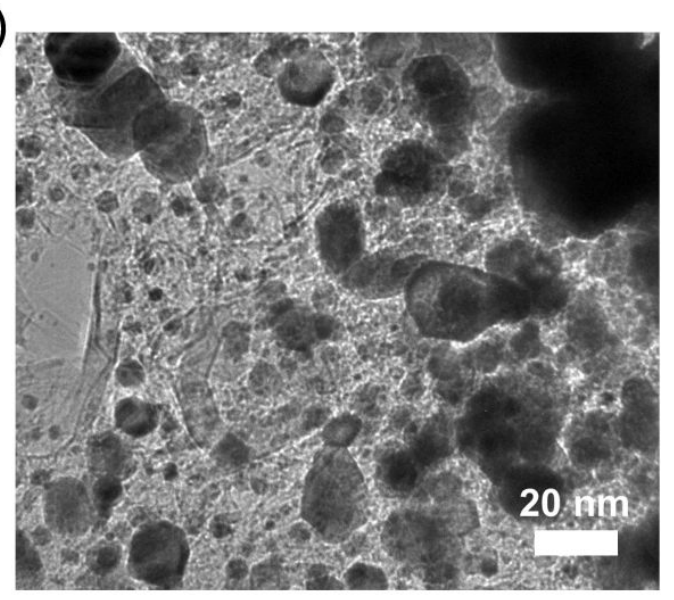

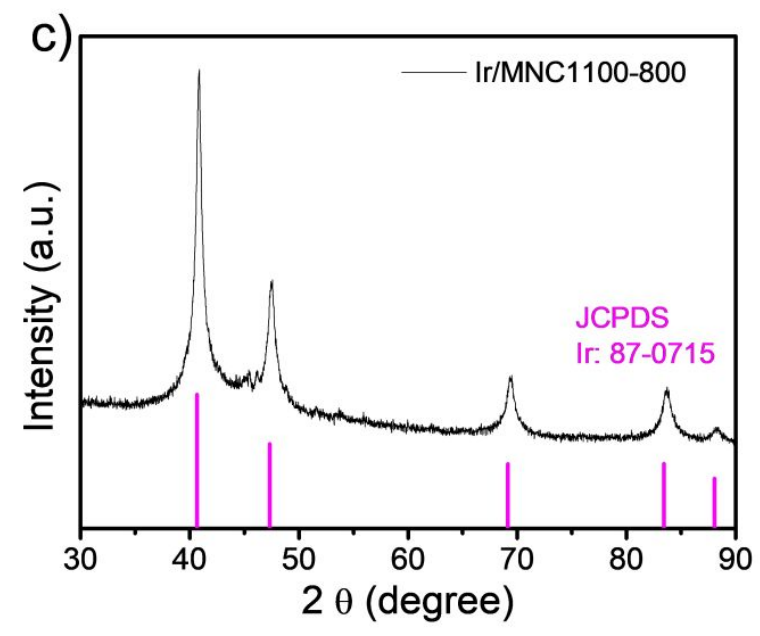

Figure S8. a,b) TEM images and c) XRD pattern of Ir/MNC1100-800. 
a)

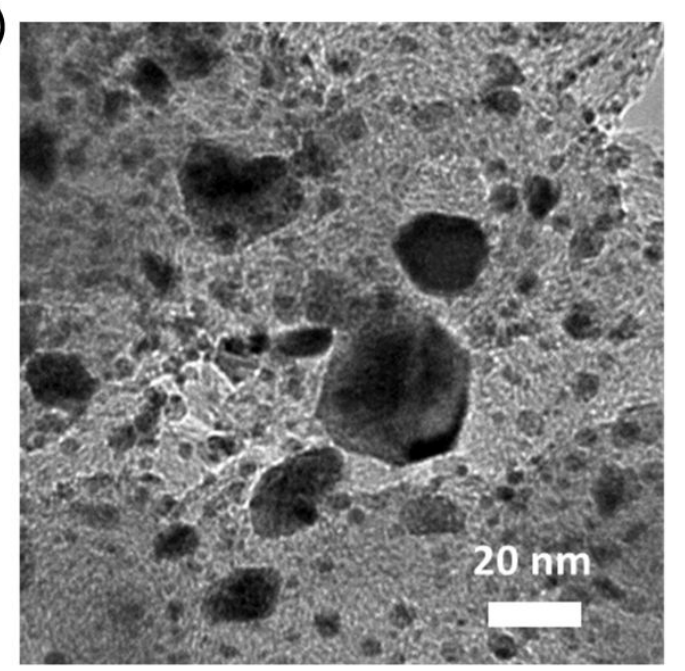

b)

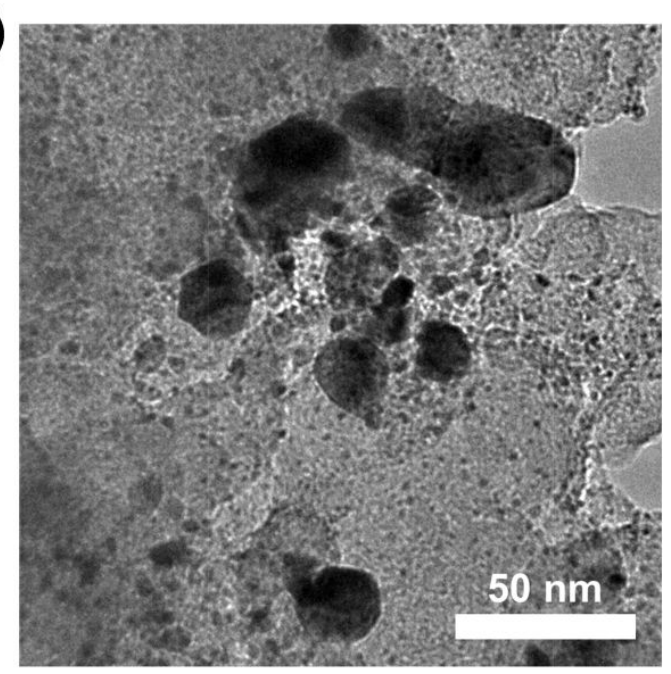

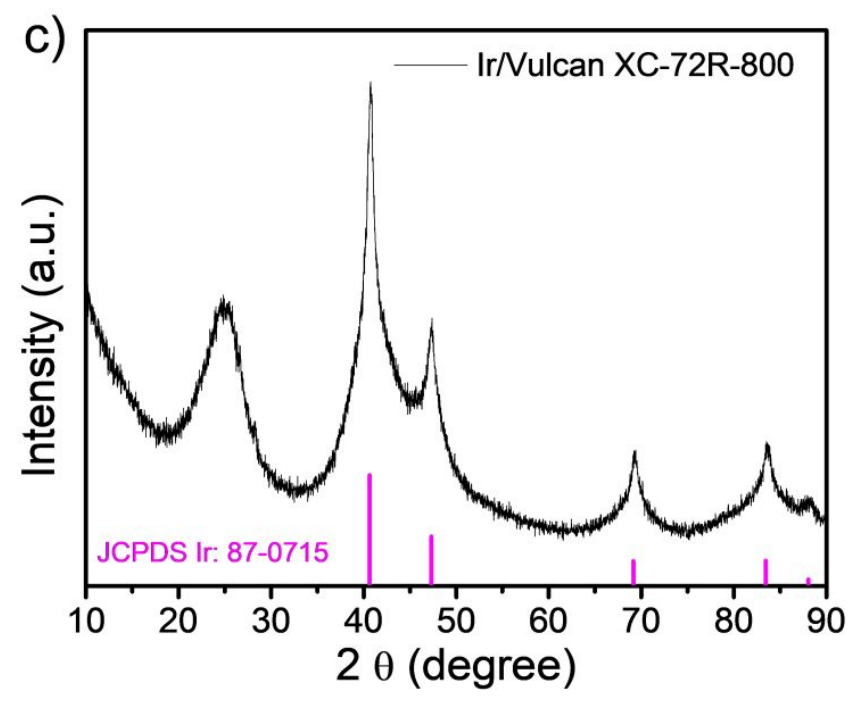

Figure S9. a, b) TEM images and c) XRD pattern of Ir/Vulcan XC-72R-800. 

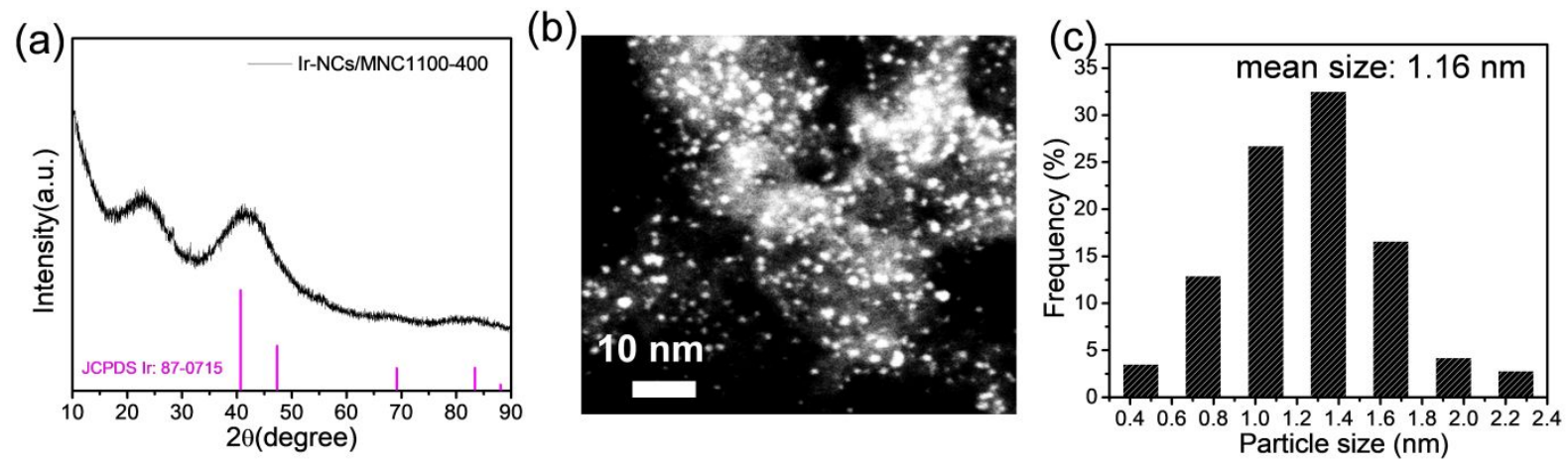

Figure S10. a) XRD pattern, b) TEM image, and (c) size distribution of Ir-NCs/MNC1100-400. 
a)

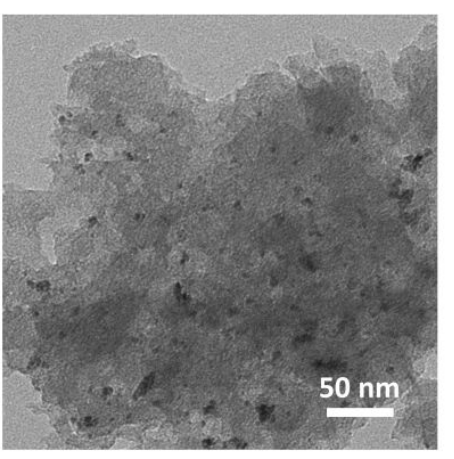

b)

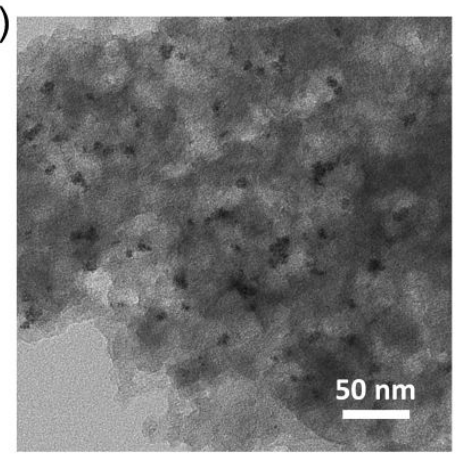

c)

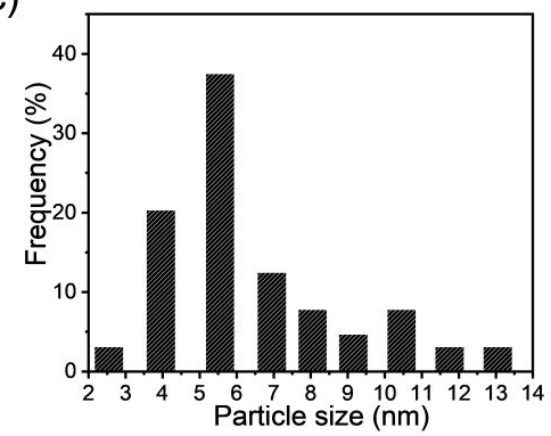

Figure S11. (a and b) TEM images and (c) size distribution of of commercial $\mathrm{Ir} / \mathrm{C}$. 


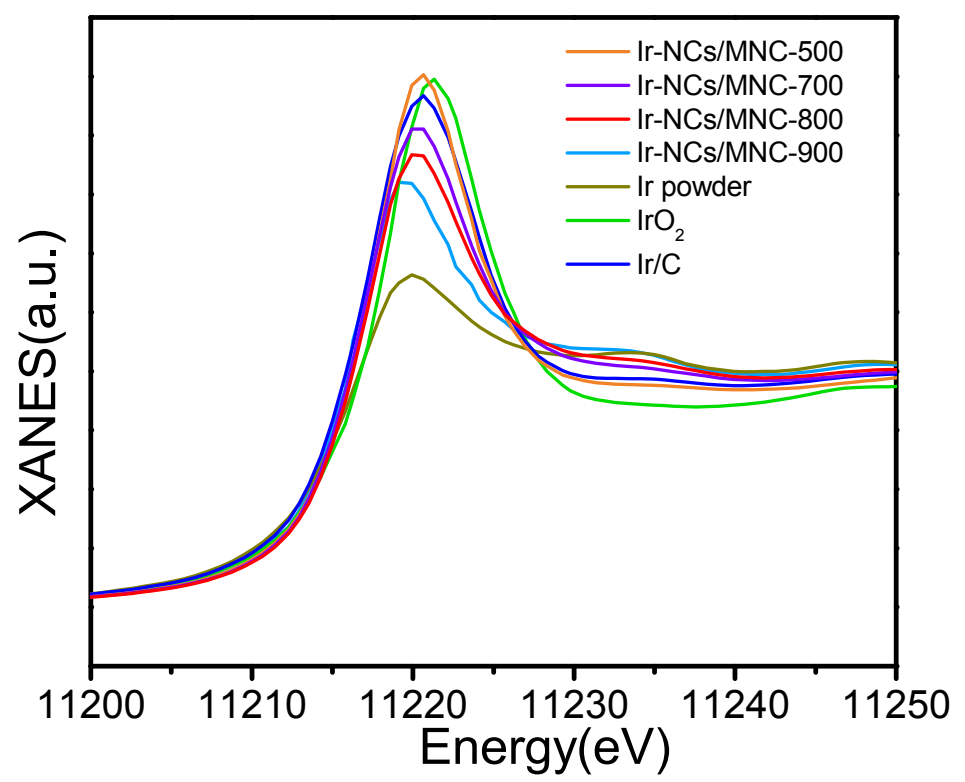

Figure S12. $\mathrm{Ir} \mathrm{L}_{3}$-edge XANES spectra of Ir-NCs/MNC, Ir powder, $\mathrm{IrO}_{2}$, and commercial $\mathrm{Ir} / \mathrm{C}$. 


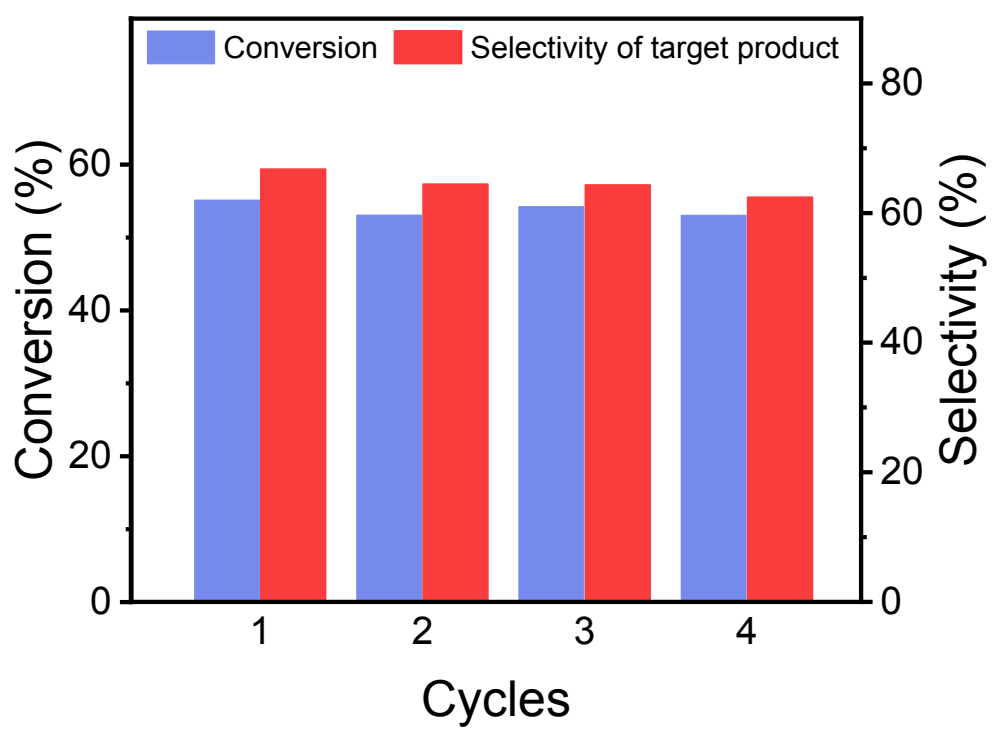

Figure S13. Recyclability of Ir-NCs/MNC-800 in the hydrogenation of $p$-chloronitrobenzene.

Reaction condition: $0.5 \mathrm{mmol}$ substrate in $1 \mathrm{ml}$ methanol, substrate/catalyst (molar) $=1000,10 \mathrm{bar}_{2}$, $10 \min , \mathrm{T}=30^{\circ} \mathrm{C}$. 
a)
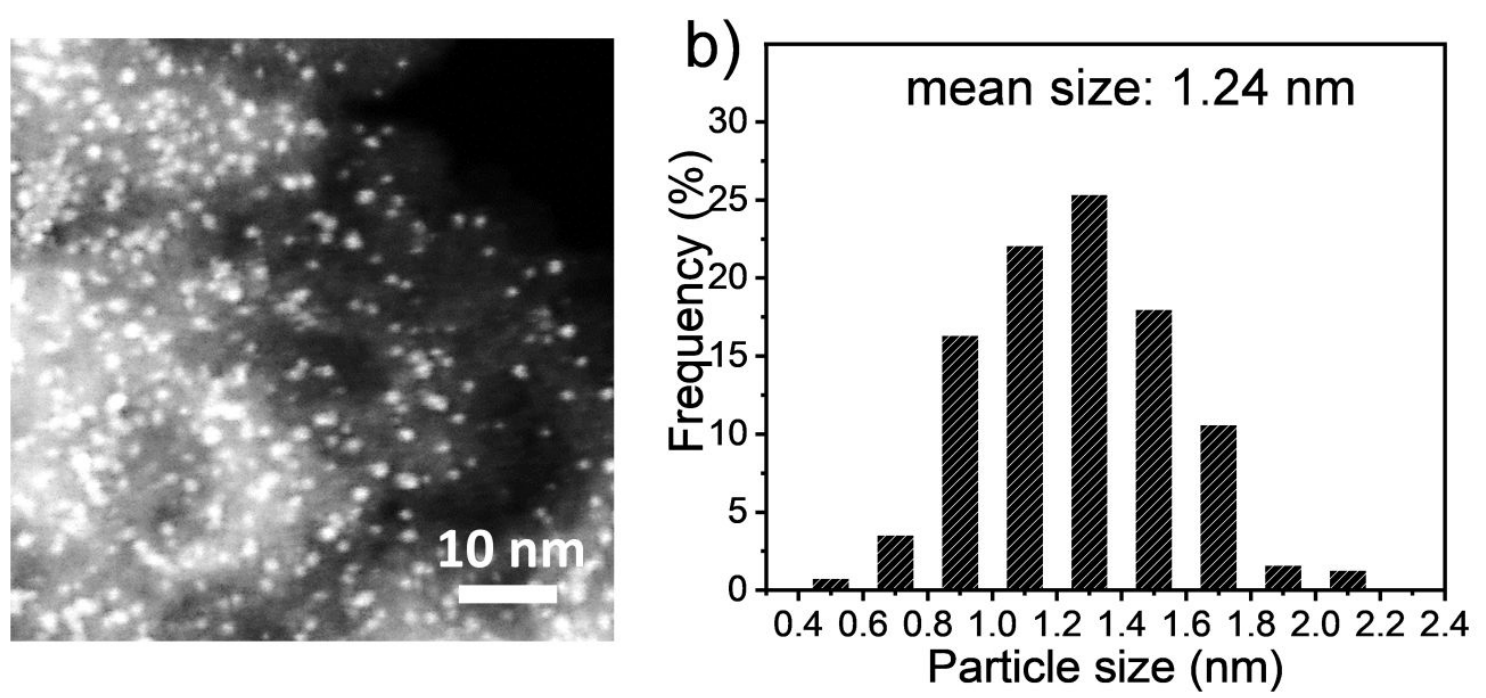

Figure S14. a) HAADF-STEM image and b) size distribution of Ir-NCs/MNC-800 after four catalytic runs. 


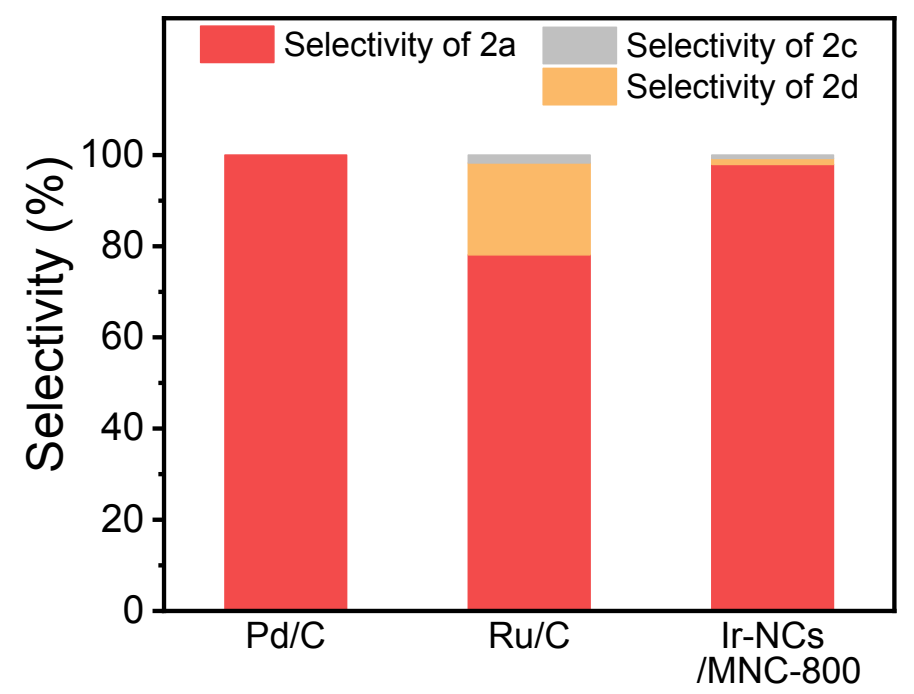

Figure S15. Selectivity for acetophenone hydrogenation at conversion of $50 \%$ by commercial $\mathrm{Ir}$ $\mathrm{NCs} / \mathrm{MNC}-800, \mathrm{Pd} / \mathrm{C}$ and $\mathrm{Ru} / \mathrm{C}$, respectively. 
Table S1. Specific surface areas and pore volume of MNC and MNC1100.

\begin{tabular}{ccc}
\hline Support & Specific surface area $\left(\mathrm{m}^{2} \mathrm{~g}^{-1}\right)$ & Pore volume $\left(\mathrm{cm}^{3} \mathrm{~g}^{-1}\right)$ \\
\hline MNC & 876 & 1.412 \\
MNC1100 & 834 & 1.514 \\
\hline
\end{tabular}


Table S2. Carbon and nitrogen contents of MCN, MCN-1100, and Ir-NCs/MNC from the EA and XPS analyses.

\begin{tabular}{cccccc}
\hline \multirow{2}{*}{ Catalyst } & \multicolumn{3}{c}{ EA content (wt\%) } & \multicolumn{2}{c}{ XPS content (at\%) } \\
\cline { 2 - 6 } MNC & $\mathrm{C}$ & $\mathrm{H}$ & $\mathrm{N}$ & $\mathrm{C}$ & $\mathrm{N}$ \\
\hline MNC1100 & 55.25 & 3.26 & 7.46 & 75.25 & 8.57 \\
Ir-NCs/MNC-500 & 53.82 & 0.46 & 0.98 & 92.63 & 1.11 \\
Ir-NCs/MNC-700 & 59.26 & 2.62 & 5.81 & 74.99 & 7.64 \\
Ir-NCs/MNC-800 & 57.14 & 2.21 & 5.62 & 75.56 & 7.57 \\
Ir-NCs/MNC-900 & 62.52 & 1.54 & 2.77 & 89.34 & 3.67 \\
\hline
\end{tabular}


Table S3. Curve-fitting results of Ir 4f XPS spectra.

\begin{tabular}{clc}
\hline \multirow{2}{*}{ Catalyst } & \multicolumn{2}{c}{ XPS content $($ at $\%)$} \\
\cline { 2 - 3 } Ir-NCs/MNC-500 & $\operatorname{Ir}(0)$ & $\operatorname{Ir}(\mathrm{IV})$ \\
Ir-NCs/MNC-700 & 67 & 33 \\
Ir-NCs/MNC-800 & 80 & 26 \\
Ir-NCs/MNC-900 & 85 & 20 \\
Ir powder & 92 & 15 \\
Ir/C & 59 & 8 \\
\hline
\end{tabular}


Table S4. Summary of the information of the commercial $\mathrm{Ir} / \mathrm{C}, \mathrm{Pt} / \mathrm{C}, \mathrm{Pd} / \mathrm{C}$ and $\mathrm{Ru} / \mathrm{C}$ catalysts.

\begin{tabular}{cccc}
\hline $\begin{array}{c}\text { Commercial } \\
\text { Catalyst }\end{array}$ & Brand & $\begin{array}{c}\text { Metal loading } \\
(\mathrm{wt} \%)\end{array}$ & $\begin{array}{c}\text { Mean particle size } \\
(\mathrm{nm})\end{array}$ \\
\hline $\mathrm{Ir} / \mathrm{C}$ & Alfa Aesar & 1 & 6.5 \\
$\mathrm{Pt} / \mathrm{C}$ & Alfa Aesar & 5 & 2.5 \\
$\mathrm{Pd} / \mathrm{C}$ & Sigma-Aldrich & 5 & 3.1 \\
$\mathrm{Ru} / \mathrm{C}$ & Sigma-Aldrich & 5 & 3.9 \\
\hline
\end{tabular}


Table S5. The effect of solvents on the selective hydrogenation of $p$-chloronitrobenzene using Ir$\mathrm{NCs} / \mathrm{MNC}^{\mathrm{a}}$

\begin{tabular}{cccc}
\hline Entry & Solvent & Conv. (\%) & Sel. (\%) \\
\hline 1 & methanol & 75 & 79.40 \\
3 & ethanol & 36 & 56.20 \\
4 & water & 31 & 80.20 \\
5 & toluene & 26 & 86.05 \\
6 & ethyl acetate & 24 & 59.31 \\
7 & THF & 23 & 52.34 \\
8 & DMF & 31 & 39.05 \\
9 & cyclohexane & 27 & 86.36 \\
\hline
\end{tabular}

Conv., conversion; Sel., selectivity.

${ }^{a}$ Reaction condition: $0.5 \mathrm{mmol} p$-chloronitrobenzene, substrate/catalyst (molar), 10 bar $\mathrm{H}_{2}, 0.5 \mathrm{~h}, \mathrm{~T}=$ $30^{\circ} \mathrm{C}$. 
Table S6 Comparison of the catalytic performances for chemoselective hydrogenation of $p$ chloronitrobenzene on various catalysts.

\begin{tabular}{ccccccccc}
\hline Catalyst & $\begin{array}{c}\text { Amount } \\
(\mathbf{m m o l} \% \\
\text { ) }\end{array}$ & $\begin{array}{c}\text { Hydrogen } \\
\text { pressure } \\
\text { (bar) }\end{array}$ & Solvent & $\begin{array}{c}\text { Temp. } \\
\left({ }^{\circ} \mathrm{C}\right)\end{array}$ & $\begin{array}{c}\text { Time } \\
(\mathbf{h})\end{array}$ & $\begin{array}{c}\text { Conv. } \\
(\mathbf{\%})\end{array}$ & $\begin{array}{c}\text { Bel. } \\
\text { (\%) }\end{array}$ & Reference \\
\hline $\mathrm{Pd} / \mathrm{CS}_{2}$ & 0.05 & 10 & ethanol & 140 & 3.5 & 100 & $>99$ & $(1)$ \\
$\mathrm{Au} / \mathrm{Sn}-\mathrm{TiO}_{2-}$ \\
123
\end{tabular}


Table S7. Hydrogenation of various substituted quinoline and N-heterocycles using Ir-NCs/MNC$800^{\mathrm{a}}$

Sel. (\%)

Conv., conversion; Sel., selectivity.

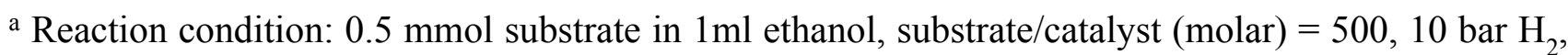
$\mathrm{T}=100^{\circ} \mathrm{C}$. 
Table S8. Hydrogenation of various substituted ketones using Ir-NCs/MNC-800a

Entry Sel. (\%)

Conv., conversion; Sel., selectivity.

a Reaction condition: $0.2 \mathrm{mmol}$ substrate in $1 \mathrm{ml}$ methanol, substrate/catalyst (molar) $=200,10$ bar $\mathrm{H}_{2}$, $\mathrm{T}=100^{\circ} \mathrm{C}$.

\section{REFENCES}

(1) Zhang, Q.; Li, K.; Xiang, Y.; Zhou, Y.; Wang, Q.; Guo, L.; Ma, L.; Xu, X.; Lu, C.; Feng, F.; Lv, J.; Ni, J.; Li, X., Sulfur-doped porous carbon supported palladium catalyst for high selective o-chloronitrobenzene hydrogenation. Applied Catalysis A: General 2019, 581, 74-81.

(2) Wang, L.; Guan, E.; Zhang, J.; Yang, J.; Zhu, Y.; Han, Y.; Yang, M.; Cen, C.; Fu, G.; Gates, B. C.; Xiao, F.-S., Single-site catalyst promoters accelerate metal-catalyzed nitroarene hydrogenation. Nat. Commun. 2018, 9, 1362.

(3) Iihama, S.; Furukawa, S.; Komatsu, T., Efficient Catalytic System for Chemoselective Hydrogenation of Halonitrobenzene to Haloaniline Using PtZn Intermetallic Compound. ACS Catal. 
2016, 6, 742-746.

(4) Li, H.-B.; Liu, L.; Ma, X.-Y., Effective Hydrogenation of Haloaromatic Nitro Compounds Catalysed by Iridium Nanoparticles Deposited on Multiwall Carbon Nanotubes. Synth. React. Inorg., Met.-Org., Nano-Met. Chem. 2016, 46, 1499-1505.

(5) Li, B.; Li, H.; Xu, Z., Experimental Evidence for the Interface Interaction in Ag/C-60 Nanocomposite Catalyst and Its Crucial Influence on Catalytic Performance. J. Phys. Chem. C 2009, $113,21526-21530$.

(6) Fan, G.-Y.; Zhang, L.; Fu, H.-Y.; Yuan, M.-L.; Li, R.-X.; Chen, H.; Li, X.-J., Hydrous zirconia supported iridium nanoparticles: An excellent catalyst for the hydrogenation of haloaromatic nitro compounds. Catal. Commun. 2010, 11, 451-455. 